\title{
Rheological blood behaviour is not related to gender in morbidly obese subjects
}

\author{
Amparo Vayáa,*, Marta Suescuna ${ }^{a}$ Eva Soláb, Marco Romagnolic and \\ Antonio Hernández-Mijares ${ }^{\mathrm{b}}$ \\ ${ }^{\mathrm{a}}$ Hemorheology and Haemostasis Unit, Service of Clinical Pathology, La Fe University Hospital, \\ Valencia, Spain \\ ${ }^{\mathrm{b}}$ Endocrinology Service, Doctor Peset University Hospital, Valencia, Spain \\ ${ }^{\mathrm{c}}$ Department of Physical Education and Sports, University of Valencia, Valencia, Spain
}

To the Editor. We have read with interest the recently published article by Wiewióra et al. [7] regarding hemorheological differences related to gender in morbidly obese subjects. In this study authors found several hemorheological differences between male and female. In this sense, males showed higher blood viscosity and higher erythrocyte deformability than females and native blood viscosity correlated with several erythrocyte aggregation parameters evaluated with the Laser-Assisted Optical Rotational Cell Analyser (LORCA) [3]. In studies with small sample sizes (18 men and 20 women) there is always the risk that differences in certain variables may appear by chance or by imbalances in the groups. Moreover, it was not discussed why the erythrocyte deformability should be higher in males.

Therefore we aimed to evaluate the rheological profile in a larger sample of morbidly obese subjects selected for bariatric surgery, to ascertain whether there are rheological differences in morbidly obese related to gender.

Before statistical testing, all continuous variables were checked for normal distribution. Those who were markedly skewed, were log transformed to improve normality.

Analytical tests were performed according to previous studies of our group [4, 5] and in accordance with the ethical guidelines for Clinical Hemorheology and Microcirculation [1]. Results are expressed in Tables 1 and 2 as mean \pm sd. $P$ values $<0.05$ were considered as statistically significant. All analyses were performed using the Statistical Package of Social Sciences (SPSS, version 11.0) for Windows.

The results of the present study revealed no differences in erythrocyte deformability, at any of the shear stresses tested when males and females were compared, in line with the homogeneity of erythrocyte indices in both groups. In this sense, Wiewióra's study did not include any information on erythrocyte indices [7], although these indices are of paramount importance when evaluating erythrocyte deformability $[2,6]$.

\footnotetext{
*Corresponding author: Amparo Vayá, MD, PhD, Hemorheology and Hemostasis Unit, Service of Clinical Pathology, La Fe University Hospital, Avda. de Campanar, 21, 46009, Valencia, Spain. Tel./Fax: +34 963862714; E-mail: vaya_amp@gva.es.
} 
Table 1

Anthropometric, lipidic and inflammatory parameters in morbidly obese males and females

\begin{tabular}{lccr}
\hline Variables & $\begin{array}{c}\text { Male } \\
(n=37)\end{array}$ & $\begin{array}{c}\text { Female } \\
(n=37)\end{array}$ & $p$ \\
\hline Age (years) & $41.8 \pm 11.7$ & $43.1 \pm 9.7$ & 0.619 \\
Weight $(\mathrm{kg})$ & $143 \pm 23$ & $120 \pm 15$ & $<0.001$ \\
BMI $\left(\mathrm{kg} / \mathrm{m}^{2}\right)$ & $44.8 \pm 4.8$ & $45.8 \pm 5.5$ & 0.407 \\
Waist $(\mathrm{cm})$ & $141.9 \pm 13.0$ & $125.9 \pm 11.2$ & $<0.001$ \\
Glucose $(\mathrm{mg} / \mathrm{dL})$ & $116 \pm 64$ & $98 \pm 27$ & 0.133 \\
HDL-cholesterol (mg/dL) & $45 \pm 15$ & $53 \pm 16$ & 0.025 \\
Triglycerides (mg/dL) & $195 \pm 237$ & $145 \pm 63$ & 0.229 \\
HbA1c $(\%)$ & $6.60 \pm 1.94$ & $5.89 \pm 1.08$ & 0.101 \\
Leptin $(\mathrm{ng} / \mathrm{mL})$ & $31.6 \pm 15.8$ & $43.1 \pm 18.2$ & 0.036 \\
Insulin $(\mu \mathrm{UI} / \mathrm{mL})$ & $28.9 \pm 17.4$ & $26.1 \pm 18.6$ & 0.383 \\
C-reactive protein $(\mathrm{mg} / \mathrm{L})$ & $7.53 \pm 6.83$ & $10.90 \pm 7.49$ & 0.029 \\
\hline
\end{tabular}

Males showed higher weight, waist circumference $(p<0.001)$ and lower HDL-cholesterol, leptin and C-reactive protein than female ( $p=0.025, p=0.036,0.029$, respectively).

Table 2

Rheological parameters in morbidly obese males and females

\begin{tabular}{lccc}
\hline Variables & Male $(n=37)$ & Female $(n=37)$ & $p$ \\
\hline Fibrinogen (mg/dL) & $378 \pm 69$ & $412 \pm 86$ & 0.082 \\
Plasma Viscosity (cP) & $1.32 \pm 0.08$ & $1.34 \pm 0.06$ & 0.257 \\
Native Blood Viscosity 230 s ${ }^{-1}(\mathrm{cP})$ & $4.41 \pm 0.45$ & $4.07 \pm 0.40$ & 0.002 \\
Corrected Blood Viscosity 230 s${ }^{-1}(\mathrm{cP})$ & $4.52 \pm 0.13$ & $4.54 \pm 0.26$ & 0.734 \\
Erythrocyte Elongation Index 12 Pa(\%) & $45.22 \pm 2.66$ & $45.31 \pm 2.92$ & 0.891 \\
Erythrocyte Elongation Index 30 Pa (\%) & $50.29 \pm 2.33$ & $50.44 \pm 2.92$ & 0.820 \\
Erythrocyte Elongation Index 60 Pa (\%) & $52.20 \pm 2.43$ & $52.01 \pm 2.78$ & 0.770 \\
Erythrocyte Aggregation at 3 s & $7.41 \pm 1.07$ & $7.81 \pm 0.88$ & 0.098 \\
Erythrocyte Aggregation Time $\left(\mathrm{s}^{-1}\right)$ & $1.53 \pm 0.36$ & $1.37 \pm 0.36$ & 0.084 \\
Erythrocyte Aggregation Index & $59.52 \pm 9.42$ & $65.07 \pm 15.08$ & 0.061 \\
\hline
\end{tabular}

In addition, a multivariate linear regression analysis which could help to clarify which parameters might be responsible for the higher erythrocyte deformability in men was not performed. Another aspect which should have been discussed is the correlation between $\mathrm{RBC}$ aggregation and native blood viscosity at $150 \mathrm{~s}^{-1}$, although they wonder about this surprising correlation, as at high shear rates these results are hard to understand, as blood viscosity at high shear rates is influenced by erythrocyte deformability [2] but not erythrocyte aggregation [8].

In the present study we have observed in morbidly obese women a non-significant tendency towards higher erythrocyte aggregability mainly associated to C-reactive protein.

We can conclude that there are no significant differences in erythrocyte deformability regarding gender in morbidly obese subjects, thus there is only a tendency in women towards a higher erythrocyte aggregation related to inflammatory parameters. 
Table 2

(continued)

\begin{tabular}{llcc}
\hline Variables & Male $(n=37)$ & Female $(n=37)$ & $p$ \\
\hline Erythrocyte Desaggregation threshold $\left(\mathrm{s}^{-1}\right)$ & $99.12 \pm 99.50$ & $103.28 \pm 83.94$ & 0.865 \\
Hematocrit (\%) & $43.80 \pm 3.48$ & $41.31 \pm 3.14$ & 0.002 \\
MCV (fL) & $87.93 \pm 4.88$ & $87.75 \pm 6.87$ & 0.903 \\
MCH (pg) & $29.44 \pm 1.99$ & $28.73 \pm 2.66$ & 0.216 \\
MCHC (g/dL) & $33.46 \pm 1.15$ & $32.99 \pm 2.00$ & 0.237 \\
\hline
\end{tabular}

Males showed higher native blood viscosity and hematocrit ( $p=0.002$, respectively) but no differences were observed when corrected blood viscosity was compared $(p=0.734)$. No differences in the erythrocyte elongation index 12, 30 and $60 \mathrm{~Pa}$ nor in the hematimetric indices (MCV, MCH, MCHC) were observed $(p>0.05)$. Although no significant differences in erythrocyte aggregation parameters were found between males and females, a tendency for higher erythrocyte aggregation was observed in females; The Pearson bivariate analysis showed that Myrenne aggregation parameters (EA1) correlated with glucose $(p<0.05)$, triglycerides $(p<0.01)$ and C-reactive protein $(p<0.01)$ whereas Sefam aggregation parameters (AI10) correlated with C-reactive protein $(p<0.05)$ and fibrinogen $(p<0.05)$ and erythrocyte aggregation time with fibrinogen $(p<0.01)$; Multiple linear regression analysis showed that $\mathrm{C}$-reactive protein was an independent predictor of EA1 values (beta coefficient: $0.312, p=0.007$ ).

\section{References}

[1] Anonymous, Ethical guidelines for publication in Clinical Hemorheology and Microcirculation, Clin Hemorheol Microcirc 44 (2010), 1-2.

[2] S. Chien, S. Usami, R.J. Dellenback and M.I. Gregersen, Blood viscosity: Influence of erythrocyte deformation, Science 157 (1967), 827-829.

[3] Y.C. Fung, Theoretical considerations of the elasticity of red cells and small blood vessels, Fed Proc 25 (1966), 1761-1772.

[4] M.R. Hardeman, P.T. Goedhart, J.G.G. Dobbe and K.P. Lettinga, Laser-assisted optical rotational cell analyser (LORCA). A new instrument for measurement of various structural hemorheological parameters, Clin Hemorheol 14 (1994), 605-618.

[5] E. Solá, A. Vayá, D. Corella, M.L. Santaolaria, F. España, A. Estellés and A. Hernández-Mijares, Erythrocyte hyperaggregation in obesity: Determining factors and weight loss influence, Obesity 15 (2007), 2128-2134.

[6] A. Vayá, R. Cámara, A. Hernádez-Mijares, M. Romagnoli, E. Solá, D. Corella and B. Laiz, Erythrocyte deformability in morbid obesity before bariatric surgery. Influence of abdominal obesity. Clin Hemorheol Microcirc 46 (2010), 313-320.

[7] A. Vayá, E. Bonet, M. Romagnoli, C. Nuñez and J. Todoli, Erythrocyte deformability in macrocytosis determined by means of ektacytometry techniques. Clin Hemorheol Microcirc 45 (2010), 27-33.

[8] T. Wetter, and H. Schmid-Schönbein, Basic rheology of mammalian blood: Factors promoting and factors interfering with fluidity of blood, Adv Exp Med Biol 164 (1984), 381-392.

[9] M. Wiewióra, K. Sosada, L. Slowinska, J. Piecuch, M. Glück, W. Zurawinski and B. Turczynski, Sex-dependent differences in rheological properties and the relation of blood viscosity to erythrocyte aggregation indices among morbidly obese patients, Clin Hemorheol Microcirc 44 (2010), 259-267. 